\title{
Cryptorchidie et infertilité masculine
}

\author{
R. Mielsset, L. Bujan*, A. Mansat*, F. Pontonnier \\ Centre de Stérilité Masculine et ${ }^{*}$ CECOS Midi-Pyrénées, CHU La Grave, Toulouse
}

\section{RESUME}

La cryptorchidie, qui fait partie des anomalies de migration du testicule, est toujours considérée comme ayant des effets très contradictoires sur la fonction exocrine des gonades mâles. Outre quelques notions nouvelles en terme d'épidémiologie et de facteurs de risque associés à la cryptorchidie, les auteurs ont brièvement revu les données de la littérature en ce qui concerne la spermatogenèse, la paternité et l'infécondité chez les hommes ayant des antécédents de cryptorchidie. Les divergences des résultats rapportés constituent une forte incitation à une approche prospective, multicentrique et multidisciplinaire des problèmes posés par les anomalies de migration du testicule.

Mots clés : cryptorchidie, paternité, spermatogenèse, infécondité

\section{INTRODUCTION}

La cryptorchidie, ou le testicule mal descen$\mathrm{du}$, est depuis longtemps l'objet d'opinions divergentes en ce qui concerne l'embryologie, la définition, l'épidémiologie, l'étiologie, la conduite à tenir et les résultats du traitement! A titre d'exemple, les enquêtes épidémiologiques ont fait apparaître des facteurs de risque aussi différents que le poids de naissance, l'âge gestationnel ou l'exposition maternelle à certaines hormones [3].
A noter cependant deux notions apparues récemment :

1. L'incidence de la cryptorchidie a nettement augmenté pendant les 30 dernières années, que ce soit en calculant les taux cumulatifs d'orchidopexies, (de $1,7 \%$ à $3,2 \%$ ) [2], ou lors de l'examen clinique des enfants à la naissance et à 3 mois, (de $0,96 \%$ à $1,85 \%$ ) [11].

2. Les anomalies de migration du testicule regroupaient jusqu'alors trois tableaux classiques, congénitaux :

a) l'ectopie ou migration du testicule hors son trajet physiologique;

b) la cryptorchidie vraie, arrêt de migration sur le trajet physiologique,

c) le testicule rétractile ou oscillant, qui se présente comme un testicule cryptorchide vrai, mais qui peut être amené en situation scrotale par manipulation et qui ne reste pas en situation scrotale [6]. Bien que très longtemps considéré comme un variant physiologique, les arguments s'accumulent dans la littérature pour considérer de plus en plus le testicule rétractile comme un variant pathologique [7].

Le tableau de ces anomalies de migration s'est cependant enrichi au cours des vingt dernières années de deux nouvelles formes que l'on peut qualifier d'acquises :

a) descente tardive: le testicule n'est pas descendu à la naissance, la descente sur- 
venant à 3 mois : dans $40 \%$ des cas, le testicule est de nouveau cryptorchide à 1 an [10].

b) testicule ascenseur: testicule scrotal à la naissance, mais qui sera ensuite retrouvé cryptorchide que ce soit vers l'âge de 1 an ou dans la période prépubertaire [6].

Quel que soit le traitement, hormonal, chirurgical ou les deux, la cryptorchidie est le seul facteur de risque établi de façon non équivoque pour le cancer du testicule, avec un risque relatif de 6 [voir 13]. La seconde conséquence néfaste de la cryptorchidie touche la fertilité, et les opinions sont divergentes quant à l'effet de la cryptorchidie sur la fertilité.

\section{CRYPTORCHIDIE ET PATERNITE}

Les hommes ayant un antécédent de cryptorchidie ont-ils moins de chance que les autres de faire un enfant ? La réponse à cette question n'est toujours pas claire. Il existe en effet un certain nombre de publications qui rapportent les taux de paternité chez les hommes ayant eu une cryptorchidie (Tableau 1). Dans les 8 études sur la cryptorchidie unilatérale $81 \%$ des hommes sont devenus pères et $51 \%$ dans les 6 études sur la cryptorchidie bilatérale. Ces résultats laisseraient entendre que la cryptorchidie unilatérale a peu d'effet sur la fertilité des hommes. Il faut cependant noter que les effectifs totaux de ces études sont faibles, que ces études sont rétrospectives et la plupart du temps sur questionnaire, avec un taux élevé de non-réponse, enfin qu'elles ont été menées sans groupe témoin.

\section{Tableau 1 : Cryptorchidie et paternité.}

Nb études Effec. totaux Pères

\begin{tabular}{llll}
\hline Unilatérale & 8 & 318 & $81 \%$ \\
Bilatérale & 6 & 128 & $51 \%$ \\
\hline
\end{tabular}

\section{CRYPTORCHIDIE ET INFECONDITE}

Il est tout aussi difficile de connaître le pourcentage d'hommes inféconds que d'hommes féconds en cas d'antécédents de cryptorchidie. Les données de la littérature font état de pourcentages d'antécédents de cryptorchidie variant de $2,5 \%$ à $9,4 \%$ (Tableau 2). En fait, les populations ne sont pas identiques, puisqu'il s'agit, soit de couples consultant en première intention pour une infécondité, soit d'hommes consultant pour une infécondité déjà reconnue dans un centre de stérilité masculine. Les biais de recrutement sont donc importants.

Tableau 2 : Cryptorchidie et infécondité.

\begin{tabular}{lcc}
\hline Auteurs & Effectifs & $\begin{array}{c}\text { Antécédents de } \\
\text { Cryptorchidies }\end{array}$ \\
\hline OMS [16] & 7273 & $2,6 \%$ \\
Abramson [1] & 200 & $2,5 \%$ \\
Yavetz et al., [17] & 8500 & $2,4 \%$ \\
Dubin \& Amelar [4] & 1294 & $4,4 \%$ \\
Ducot et al., [5] & 394 & $9,4 \%$ \\
Mieusset et al., [14] & 1014 & $9,4 \%$ \\
\hline
\end{tabular}

\section{CRYPTORCHIDIE ET SPERMATOGENESE}

De nombreuses études histologiques ont montré une détérioration progressive qui survenait très précocement, pour certains auteurs dès la première année après la naissance : le nombre moyen de cellules germinales dans le testicule cryptorchide est le même que dans le testicule normal à 6 mois, mais diminué à partir de 12 mois [9]. Est rapporté aussi une diminution fréquente du nombre de cellules de Leydig [12]. La croissance du testicule cryptorchide est perturbée, le volume testiculaire étant diminué chez $45 \%$ des enfants $[9,12]$. Chez les hommes inféconds ayant un antécédent de cryptorchidie (traitée), le testicule anciennement cryptorchide est de volume toujours inférieur au controlatéral scrotal, 
mais ce dernier est lui-même plus petit que le testicule correspondant chez les hommes féconds sans antécédent de cryptorchidie [14]. En ce qui concerne le sperme, une revue de la littérature de Chilvers \& Pike [3] retrouvait une azoospermie dans 14 à $42 \%$ pour les cryptorchidies uni ou bilatérales et un taux d'oligospermie à $31 \%$ dans les deux cas (Tableau 3). De plus, la qualité des spermatozoïdes est moindre avec une réduction de la mobilité et de la morphologie normale des spermatozoïdes [14].

Tableau 3 : Cryptorchidie et spermatogénèse.

\begin{tabular}{lcc}
\hline $\begin{array}{l}\text { Numération } \\
(106 / \mathrm{ml})\end{array}$ & 0 & 0,1 à 20 \\
$\begin{array}{l}\text { Unilatérale } \\
(\mathrm{n}=600)\end{array}$ & $14 \%$ & $31 \%$ \\
$\begin{array}{l}\text { Bilatérale } \\
(\mathrm{n}=331)\end{array}$ & $42 \%$ & $31 \%$ \\
\hline
\end{tabular}

D'après Chilvers \& Pike [3].

\section{CRYPTORCHIDIE ET TEMPÉRATURE TESTICULAIRE}

Comme nous l'avons largement développé dans un numéro récent de la revue ANDROIOGIE [13], nous ne ferons que rappeler ici que la migration physiologique du testicule en situation scrotale est associée à une réduction de l'environnement thermique du testicule. En cas d'arrêt de migration (cryptorchidie par exemple), la température du testicule est plus élevée (en moyenne $1^{\circ} \mathrm{C}$ ) que celle du testicule controlatéral normalement descendu (Figure 1). Il n'existe aucune donnée chez l'homme permettant d'affirmer que l'élévation de la température du testicule cryptorchide est responsable des perturbations de la spermatogenèse classiquement observées; nous ne savons pas non plus si l'abaissement du testicule cryptor- chide en situation scrotale amène une normalisation de la température du testicule anciennement cryptorchide. Chez les adultes consultant pour infécondité masculine et ayant un antécédent de cryptorchidie traitée, la fréquence d'une élévation anormale de la température scrotale $(45 \%)$ est plus importante que les hommes inféconds sans antécédent de cryptorchidie $(30 \%)$. Cette élévation anormale de la température scrotale est associée à une réduction des volumes testiculaires, de la quantité et de la qualité des spermatozoïdes produits (c'est-à-dire de la spermatogenèse), ainsi qu'à une fertilité moindre que chez les inféconds avec antécédents de cryptorchidie mais ayant des températures scrotales normales [voir 13].

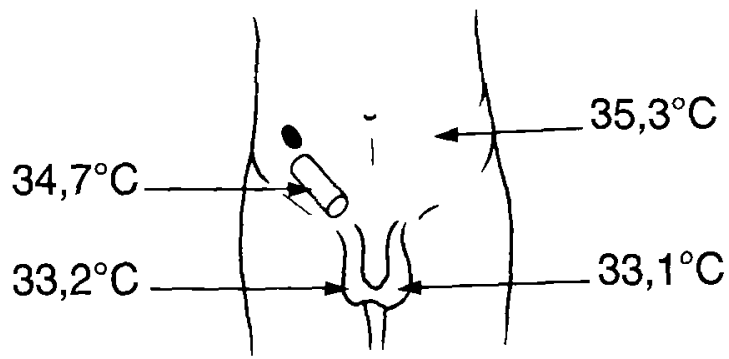

Figure 1 : Migration du testicule et température. Le testicule, originellement situé dans l'espace Bogros $\left(35,3^{\circ} \mathrm{C}\right)$ va, grâce à sa migration, atteindre la cavité scrotale (dont la température en l'absence de testicule est à $33,1^{\circ} \mathrm{C}$ ) $\grave{a}$ l'intérieur de laquelle sa température sera de $33,2^{\circ} \mathrm{C}$. En cas d'arrêt de migration dans le canal inguinal, sa température sera supérieure, $34,7^{\circ} \mathrm{C}$ [13].

En conclusion de ce chapitre sur les relations entre cryptorchidie et température testiculaire, on peut fortement regretter que la notion physiologique de thermorégulation du testicule ne soit pas prise en compte. Enfin, chez les hommes adultes inféconds ayant un antécédent de cryptorchidie il est clair que chez $45 \%$ d'entre eux l'existence d'une température scrotale représente un facteur de risque supplémentaire pour leur spermatogenèse et leur fécondité. 


\section{CONCLUSIONS}

De cette courte revue sur cryptorchidie et infécondité masculine, il ressort peu d'éléments de certitude mais de nombreuses questions, parmi lesquelles nous retiendrons entre autre :

- le problème de définition des anomalies de migration des testicules, définition sous-entendant diagnostic et impliquant conduite à tenir;

- l'absence de notion précise quant à l'incidence de la cryptorchidie en France, avec ces deux conséquences potentielles: cancer du testicule et infécondité;

- l'absence de données claires concernant le retentissement de la cryptorchidie sur la fécondité de l'homme.

Il paraît peu probable d'obtenir des réponses à ces questions si ce n'est à travers une approche qui soit multidisciplinaire, multicentrique et prospective.

\section{BIBLIOGRAPHIE}

1. ABRAMSSON L., DUCHEK M., LUNDGREN B. : Conception rate for infertile couples. Scand. J. Urol. Nephrol. 1989, $23:$ 165-171.

2. CHILVERS C., DUDLEY N.E., COUGH M.H., JACKSON M.B., PIKE M.C. : Undescended testis: the effect of treatment on subsequent risk of subfertility and malignancy. J. Pediatr. Surg. 1986, 21 : 691-696.

3. CHILVERS C., PIKE M.C. : Epidemiology of undescended testis. In Oliver R.T.D., Blandy J.P., HopeStone H.F. eds. Urological and Genital Cancers. Oxford, Blackwell Scientific, 1989 : 306-321.

4. DUBIN L., AMELAR R.D. : Etiologic factors in 1294 consecutive cases of male infertility. Fertil. Steril. 1977, $22: 469-474$.

5. DUCOT B., SPIRA A., FENEUX D., JOUANNET $P$. : Male factors and the likelyhood of pregnancy in infertiles couples. II. Study of clinical characteristics - practical consequences. Int. J. Androl. $1988,11: 395-404$.

6. GOH D.W., HUSTON J.M. : Is the retractile testis a normal physiological variant or an anomaly that requires active treatment? Pediatr. Surg. Int., $1992,7: 249-252$.
7. GOH D.W., HUSTON J.M. : The retractile testis time for a reappraisal. J. Pediatr. Child. Health, 1993, $29:$ 407-408.

8. HADZISELIMOVIC F., HERZOG B., BUSER M. : Development of cryptorchid testes. Eur. J. Pediatr., 1987, 146 (suppl 2) : 508-512.

9. ITO H., KATAUMI Z., YANAGI S. et al : Changes in the volume and histology of retractile testes in prepubertal boys. Int. J. Androl., 1986, 9 : 161-169.

10. JOHN RADCLIFFE HOSPITAL CRYPTORCHIDISM STUDY GROUP : Boys with late descending testes, the source of patients with "retractile" testes undergoing orchidopexy? Br. Med. J., 1986, $293: 789-790$.

11. JOHN RADCLIFFE HOSPITAL CRYPTORCHIDISM STUDY GROUP : Cryptorchidism: a prospective study of 7500 consecutive male births, 1984-8.Arch. Dis. Child., 1992, 67 : 892-899.

12. KOGAN S.J. : Fertility in cryptorchidism. An overview in 1987. Eur. J. Pediatr., 1987, 146 (suppl 2) : 521-524.

13. MIEUSSET R. : Cryptorchidie et cancer du testicule. Andrologie, 1994, 2 : 190-196.

14. MIEUSSET R., BUJAN L., MASSAT G., MANSAT A., PONTONNIER F. : Clinical and biological characteristics of infertile men with a history of cryptorchidism. Hum. Reprod., 1995, $10:$ 613-619.

15. MIEUSSET R. : Cryptorchidie et température testiculaire. Andrologie, 1995, 3: 317-325.

16. OMS: The influence of varicocele on parameters of fertility in a large group of men presenting to infertility clinics. Fertil. Steril. 1992, $57: 1289$ 1293.

17. YAVETZ H., HARASH B., YOGEV L., HOMONNAI Z.T., PAZ G. : Fertility of men following inguinal hernia repair. Andrologia 199i, $23: 443-446$.

\section{ABSTRACT}

Cryptorchidism and male infecondity

\section{R. Mieusset, L. Bujan, A. Mansat, F. PonTonNier}

Cryptorchidism is one of the various forms of testicular maldescent. Cryptorchidism is still reported to have discrepant effects upon the exocrine function of the male gonade. Besides some new information about epidemiology and risck factors associated with cryptorchidism, data from the literatu- 
re are reviewed concerning spermatogenesis, fatherhood and infertility in men with a history of cryptorchidism. The discrepancy of the results is such important that only prospective and multicentric studies involving various experts seem to be appropriate to approach the complex problems arising from the maldescended testis.

Key words : Cryptrochidism, fatherhood, spermatogenesis, infertility. 